Bull. Austral. Math. Soc.

VOL. 49 (1994) [353-364]

\title{
PINCHING THEOREMS FOR TEARDROPS AND FOOTBALLS OF REVOLUTION
}

\author{
JOSEPH E. BORZELLINO
}

\begin{abstract}
We give explicit optimal curvature pinching constants for the Riemannian $(p, q)$ football orbifolds under the assumption that they are realised as surfaces of revolution in $\mathbb{R}^{3}$. We show that sufficiently pinched sectional curvature assumptions imply that a $(p, q)$-football must be good.
\end{abstract}

\section{INTRODUCTION}

In this paper, a first step is made in answering a question posed by Thurston in [5] where he asks for the best pinching constant for Riemannian metrics on the so-called teardrop and football orbifolds. Recall that given integers $1 \leqslant p \leqslant q, a(p, q)$-football $F$ is an orbifold whose underlying space is $S^{2}$, and whose singular locus consists of two points. An open neighbourhood of one of these points is modelled on the quotient of the unit disc $D^{2} \subset \mathbb{R}^{2}$ by $\mathbb{Z}_{p}$, where $\mathbb{Z}_{p}$ acts on $D^{2}$ by rotation about 0 . The other point is similarly modelled on the quotient of $D^{2}$ by a $\mathbb{Z}_{q}$ cyclic action fixing 0 . If $p=1$, then $F$ is commonly referred to as a teardrop, while if $q=1$, then $p=1$, and $F$ is the standard sphere $S^{2}$ (that is, the orbifold whose underlying space is $S^{2}$, and whose singular locus is empty). For convenience, we regard teardrops as special cases of footballs and refer to all such orbifolds $F$ as footballs. Since we are interested in studying Riemannian metrics on orbifolds, we assume that the complements of the singular loci are smooth Riemannian manifolds, and that neighbourhoods of the singular points are isometric to $\left(D^{2}, g\right) / \mathbb{Z}_{r}$, where $g$ is some smooth Riemannian metric on $D^{2}$ and $\mathbb{Z}_{r}$ acts by isometries on $D^{2}$ fixing a single point. This data is sufficient to equip our orbifolds with a Riemannian structure. For more detailed information, the reader should consult [5]. A more Riemannian viewpoint is taken in both [1] and [2].

We say that a Riemannian orbifold is good if it arises as a global quotient $M / G$, where $M$ is a Riemannian manifold, and $G$ is a group of isometries acting (properly) discontinuously on $M$. Riemannian orbifolds that do not arise in this way are called bad. In [5], it is shown that a $(p, q)$-football is good if and only if $p=q$. In [2], it is proven that a $n$-dimensional complete Riemannian orbifold with Ricci curvature at

Received 31st May, 1993

The author would like to thank Wayne Rossman for some useful discussions regarding this work.

Copyright Clearance Centre, Inc. Serial-fee code: 0004-9729/94 \$A2.00+0.00. 
least $(n-1)$ has diameter at most $\pi$, and if the diameter equals $\pi$, the orbifold must be good. In particular, a football with sectional curvature at least 1 and diameter $\pi$, is good.

For our purposes, we consider only those those orbifolds which arise as surfaces of revolution in $\mathbb{R}^{3}$. Given the results above, it seems natural to consider the following interpretations of Thurston's problem.

Theorem 1. Let $F$ be a smooth Riemannian $(p, q)$-football that arises as a surface of revolution in $\mathbb{R}^{3}$ with diameter $\pi$. Assume $1 \leqslant p \leqslant q$. Then there exists an explicit constant $\delta_{0}(p, q) \geqslant 0$, depending only on $p$ and $q$, such that if $0 \leqslant \sec (F) \leqslant$ $1+\delta_{0}$, then $p=q$. In other words, $F$ is a good football. Moreover, $\delta_{0}$ is optimal. This means that if $\delta_{0}$ is replaced by $\delta>\delta_{0}$, then there exists a $(p, q)$-football of revolution $F$ with $p \neq q$ such that $0 \leqslant \sec (F)<1+\delta$. The explicit formula for $\delta_{0}$ is:

$$
\delta_{0}=\frac{1}{\pi^{2}}\left[\arccos \left(-\frac{p}{q}\right)+\sqrt{\left(\frac{q}{p}\right)^{2}-1}\right]^{2}-1
$$

THEOREM 2. Let $F$ be a smooth Riemannian $(p, q)$-football that arises as a surface of revolution in $\mathbb{R}^{3}$. Assume $1 \leqslant p \leqslant q$. Then there exists an explicit constant $\varepsilon_{0}(p, q) \geqslant 0$, depending only on $p$ and $q$, such that if $1 \leqslant \sec (F) \leqslant 1+\varepsilon_{0}$, then $p=q$. In other words, $F$ is a good football. Moreover, $\varepsilon_{0}$ is optimal. This means that if $\varepsilon_{0}$ is replaced by $\varepsilon>\varepsilon_{0}$, then there exists a $(p, q)$-football of revolution $F$ with $p \neq q$ such that $1 \leqslant \sec (F)<1+\varepsilon$. The explicit formula for $\varepsilon_{0}$ is:

$$
\varepsilon_{0}=\left(\frac{q}{p}\right)^{2}-1
$$

REMARK 3. It is clear that the formulas for $\delta_{0}$ and $\varepsilon_{0}$ depend only on the ratio $p q^{-1}$. This is to be expected since a $(p, q)$-football can be regarded as an $k$-fold Riemannian covering orbifold of a $(k p, k q)$-football, and hence both are locally isometric away from the singular set. In the last section of this paper, we compute some values of $\delta_{0}$ for specific values of $p q^{-1}$.

EXAMPLE 4. A smooth $(2,5)$-football $F$ of revolution with diameter $\pi$ cannot admit a metric whose sectional curvature satisfies $0 \leqslant \sec (F) \leqslant 1.85$, by Theorem 1 .

EXAMPLE 5. A smooth $(2,5)$-football $F$ of revolution cannot admit a metric whose sectional curvature satisfies $1 \leqslant \sec (F) \leqslant 6.25$, by Theorem 2 .

A good football of diameter $\pi$ always admits a metric of constant curvature 1 , by realising it as an appropriate quotient of $\left(S^{n}\right.$, can), the sphere of constant curvature 1. It is shown in the proof of Theorem 1 (respectively, Theorem 2) that $\delta_{0}=0$ (respectively, $\varepsilon_{0}=0$ ) implies that $p=q$. Hence we have the following corollary. 
Corollary 1. Let $F$ be as above in Theorem 1 (respectively, Theorem 2). Then the following are equivalent:

(i) $\delta_{0}=0$ (respectively, $\varepsilon_{0}=0$ );

(ii) $p=q$.

One might also consider the related question: Suppose $F$ is a smooth Riemannian $(p, q)$-football with diameter $\pi$. Does there exist $\mu_{0} \geqslant 0$, such that if $\sec (F) \geqslant 1-\mu_{0}$, then $p=q$ ? If $\mu_{0}=0$, then the results in [2] mentioned above imply that $p=q$. However, the next result shows that in fact $\mu_{0}=0$ is optimal.

THeOREM 7. There exists a sequence $\left\{F_{n}\right\}$ of bad $(p, q)$-footballs $F_{n}$ such that $\operatorname{diam} F_{n}=\pi$ and $1-1 / n \leqslant \sec \left(F_{n}\right)$.

The classification of bad 2-dimensional orbifolds [5], shows that if $O$ is any compact 2-dimensional bad orbifold that admits a metric with nonnegative curvature then $O$ has an orientable orbifold double covering which is either a football or teardrop. Such bad orbifolds are either teardrops, footballs, or $(p, q)$-hemispheres: these are orbifolds whose underlying space is $D^{2}$ and whose singular locus is modelled locally on quotients of $D^{2}$ by the dihedral group $D_{n}$, the group of order $2 n$ generated by reflections in two lines which intersect at an angle of $\pi / n$. The singular locus of the quotient $D^{2} / D_{n}$, is commonly referred to as a corner reflector of order $n$. More simply, take a $(p, q)$-football (or teardrop) and reflect across the "equator" containing the two singular points. The resulting quotient is a $(p, q)$-hemisphere. If $F$ is a compact 2-dimensional bad Riemannian orbifold whose orientable orbifold double covering is a football of revolution, we shall call $F$ an orbifold of revolution. With this in mind, one gets the immediate corollary to Theorems 1 and 2:

COROLLARY 8. Theorems 1 and 2 remain valid if $F$ is any compact 2-dimensional bad Riemannian orbifold of revolution.

One last result is that the proofs of Theorems 1 and 2 do not use the fact that $p$ and $q$ are integers. Hence, Theorems 1 and 2 remain valid for cone-footballs. These are footballs whose singular locus is modelled on a cone of (possibly irrational) angle $\alpha$ with $0<\alpha \leqslant 2 \pi$.

Corollary 9. Theorems 1 and 2 remain valid if $F$ is only assumed to be a $(p, q)$-cone-football of revolution.

The main tool in proving Theorem 1 and Theorem 2 will be the Sturm Comparison Theorem. The explicit solvability of the comparison differential equation makes it possible to compute the optimal pinching constants.

The next few sections of this paper will be devoted to the setup and proof of Theorems 1 and 2 . To fix notation, we shall first recall the relevant facts about surfaces 
of revolution in $\mathbb{R}^{3}$, and give a precise statement of the Sturm Comparison Theorem that will be sufficient for our needs.

\section{SURFACES OF REVOLUTION AND THE STURM COMPARISON THEOREM}

Although what is done in this section is elementary, to achieve the goal of computing explicit pinching constants requires us to catalog the necessary formulas to be used.

\section{Surfaces of Revolution and Flat Cones.}

Let $\gamma=(x(t), y(t)): I=[0, b] \rightarrow \mathbb{R}^{2}$ be a smooth arc-length parametrised curve in the plane with $y(t)>0$ on $(0, b)$ and $y(0)=y(b)=0$. Recall that the surface of revolution obtained by revolving the curve $\gamma$ about the $x$-axis in $\mathbb{R}^{3}$ is given by the $\operatorname{map} f: I \times \mathbb{R} \rightarrow \mathbb{R}^{3}$

$$
(t, \theta) \mapsto(x(t), y(t) \cos \theta, y(t) \sin \theta)
$$

with metric $g=d t^{2}+[y(t)]^{2} d \theta^{2}$. Alternatively, this surface may be regarded as the metric completion of the Riemannian warped product $(0, b) \times_{y(t)} S^{1}$. The map $f$ restricted to $\theta=\theta_{0}=$ constant is called a meridian and the map $f$ restricted to $t=$ $t_{0}=$ constant is called a parallel. Since the metric $g$ depends only on $t, g$ is rotationally symmetric, and thus the sectional curvature $K$ at a point $(t, \theta)$ depends only on the value of $t$. A standard computation shows that the curvature $K(t)=-y^{\prime \prime} / y$. See for example [3, page 238].

Definition 10: A flat cone of type $\alpha$ is the flat metric space obtained by identifying the two edges of an infinite wedge of (interior) angle $\alpha$ in $\mathbb{R}^{2}$. See Figure 1.

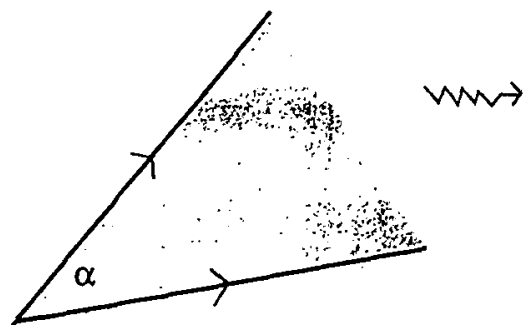

wedge in $\mathrm{R}^{2}$

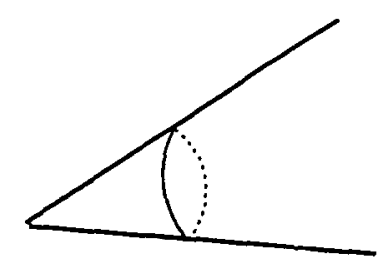

cone in $\mathrm{R}^{3}$

Figure 1

As a surface of revolution in $\mathbb{R}^{3}$, a flat cone can be realised by rotating the half-line $y(t)=(\tan \theta) t, t \geqslant 0$ about the $x$-axis in $\mathbb{R}^{3}$, where $0 \leqslant \theta \leqslant \pi / 2$ is the angle that the line $y$ makes with the $x$-axis in $\mathbb{R}^{2}$. An arc-length parametrisation of $y$ is given by $\sigma(t)=(t \cos \theta, t \sin \theta)$. The (2-dimensional) volume of $B(v, R)$, the metric ball of radius $R$ centred at the vertex $v$ of the cone, is given by

$$
\text { Vol } B(v, R)=2 \pi \int_{0}^{R} t \sin \theta d t=\pi R^{2} \sin \theta .
$$


On the other hand, the same metric ball $B(v, R)$ on a flat cone of type $\alpha$, has volume Vol $B(v, R)=(\alpha / 2 \pi)\left(\pi R^{2}\right)=\alpha R^{2} / 2$. Equating the two expressions for $\operatorname{Vol} B(v, R)$ yields

$$
\theta=\arcsin \left(\frac{\alpha}{2 \pi}\right) \text {. }
$$

Footballs As Surfaces of Revolution.

We now exhibit $(p, q)$-footballs as surfaces of revolution. Suppose that $\gamma=$ $(x(t), y(t))$ is a smooth profile curve for a $(p, q)$-football $F$ of diameter $D$ with curvature $K(t) \geqslant 0$. Note that by definition of Riemannian football the singular points of $F$ must correspond to $\gamma(0)$ and $\gamma(D)$. Also the singular points must realise the diameter $D$. To see this, let $x_{1}, x_{2}$ be two points such that $d\left(x_{1}, x_{2}\right)=D$. Let $d(\gamma(0), \gamma(D))=C$. Then by the triangle inequality

$$
\begin{aligned}
d\left(\gamma(0), x_{1}\right)+d\left(\gamma(0), x_{2}\right) \geqslant d\left(x_{1}, x_{2}\right) & =D \\
d\left(\gamma(D), x_{1}\right)+d\left(\gamma(D), x_{2}\right) \geqslant d\left(x_{1}, x_{2}\right) & =D .
\end{aligned}
$$

However, note that $d\left(\gamma(0), x_{i}\right)+d\left(x_{i}, \gamma(D)\right)=C$ since meridians are minimising geodesics. Adding the two previous inequalities then implies that $C \geqslant D$. Since $D$ is the diameter of $F$, we must have $d(\gamma(0), \gamma(D))=D$.

Thus, in the notation of the previous section, we have, $I=[0, D], y(t)>0$ on the open interval $(0, D)$, and $y(0)=y(D)=0$. Furthermore, the curvature condition implies that $y^{\prime \prime} \leqslant 0$ for $t \in(0, D)$. We are assuming that $1 \leqslant p \leqslant q$. Note that the tangent line to $\gamma$ at $t=0$ must sweep out a flat cone (opening in the positive $x$ direction with vertex $(0,0,0))$ of type $2 \pi / p$, and that the tangent line to $\gamma$ at $t=D$ is a flat cone (opening in the negative $x$ direction with vertex $(D, 0,0)$ ) of type $2 \pi / q$. Thus we have

$$
\begin{array}{r}
\gamma^{\prime}(0)=\tan \left(\arcsin \left(\frac{1}{p}\right)\right)=\left(p^{2}-1\right)^{-1 / 2} \stackrel{\text { def }}{=} \xi_{p} \\
-\gamma^{\prime}(\pi)=\tan \left(\arcsin \left(\frac{1}{q}\right)\right)=\left(q^{2}-1\right)^{-1 / 2} \stackrel{\text { def }}{=} \xi_{q}
\end{array}
$$

Note that $\xi_{p} \geqslant \xi_{q}$. In the special case where $p=1$, we regard $\xi_{p}=+\infty$, likewise $\xi_{q}=+\infty$ if $q=1$ also. Since we have assumed that $\gamma$ is parametrised by arc-length, we have

$$
x(t)=\int_{0}^{t} \sqrt{1-\left[y^{\prime}(s)\right]^{2}} d s .
$$

In order for $x(t)$ to be defined we must have $\left|y^{\prime}(t)\right| \leqslant 1$. We shall show this shortly, but for now assume that $x(t)$ is well-defined. Now

$$
\gamma^{\prime}(0)=\frac{y^{\prime}(0)}{x^{\prime}(0)}=\frac{y^{\prime}(0)}{\sqrt{1-\left[y^{\prime}(0)\right]^{2}}}, \quad \text { and similarly } \quad \gamma^{\prime}(D)=\frac{y^{\prime}(D)}{\sqrt{1-\left[y^{\prime}(D)\right]^{2}}}
$$


A simple analysis of the function $h(z)=z / \sqrt{1-z^{2}}$ shows that $h$ is monotone increasing on the interval $(-1,1)$ and the range of $h$ is $\mathbb{R}$. The inverse function is $h^{-1}(z)=$ $z / \sqrt{1+z^{2}}: \mathbb{R} \rightarrow(-1,1)$. Thus, in order for our profile curve to have the correct initial and terminal slopes, we need

$$
\begin{gathered}
y^{\prime}(0)=h^{-1}\left(\xi_{p}\right)=\frac{\xi_{p}}{\sqrt{1+\xi_{p}^{2}}}=p^{-1} \\
y^{\prime}(D)=h^{-1}\left(-\xi_{q}\right)=\frac{-\xi_{q}}{\sqrt{1+\xi_{q}^{2}}}=-q^{-1}
\end{gathered}
$$

Summarising our analysis, we have shown that if $\gamma=(x(t), y(t))$ is the profile curve for a $(p, q)$-football of revolution of diameter $D$, and with curvature $K(t) \geqslant 0$, then the following conditions must be satisfied for the function $y(t)$ :

$$
\begin{aligned}
& \text { (i) } y^{\prime \prime}+K(t) y=0 \text { for } t \in[0, D] \text {, } \\
& \text { (ii) } y>0 \text { for } t \in(0, D) \\
& \text { (iii) } y(0)=y(D)=0 \\
& \text { (iv) } y^{\prime}(0)=p^{-1}, y^{\prime}(D)=-q^{-1} \\
& \text { (v) } y^{\prime \prime} \leqslant 0
\end{aligned}
$$

Condition (v) implies that $K(t) \geqslant 0$ and that $y^{\prime}(t)$ is monotone non-increasing. Since $1 \geqslant p^{-1}=y^{\prime}(0) \geqslant q^{-1}=-y^{\prime}(D)>0$, it follows that $y^{\prime 2} \leqslant 1$, and hence that $x(t)=\int_{0}^{t} \sqrt{1-\left[y^{\prime}(s)\right]^{2}} d s$ is well-defined.

\section{The Sturm Comparison Theorem.}

We shall need the following version of the Sturm Comparison Theorem. See [4, p.333] for a proof.

THEOREM 11. Let $f, h$ be two continuous functions satisfying $f(t) \leqslant h(t)$ for all $t$ in an interval $I$, and let $\phi, \eta$ be two functions satisfying the following differential equations on $I$ :

$$
\begin{gathered}
\phi^{\prime \prime}+f \phi=0 \\
\eta^{\prime \prime}+h \eta=0
\end{gathered}
$$

Assume that $\phi \not \equiv 0$, and let $a, b \in I$ be two consecutive zeros of $\phi$. Assume that $\eta(a)=\phi(a)=0$ and that $\eta^{\prime}(a)=\phi^{\prime}(a)>0$. If $\tau$ is the smallest zero of $\eta$ in $(a, b]$, then

$$
\phi(t) \geqslant \eta(t), \quad \text { for } a \leqslant t \leqslant \tau
$$

with equality for some $t$ if and only if $f=h$ on $[a, t]$. 


\section{EXAMPLES.}

In this section we construct the examples of Theorem 7.

Proof of Theorem 7: We use the notation and setup as in $\left({ }^{*}\right)$. Fix $p \neq q$. Consider curves of the following type:

$y_{1}^{(n)}(t)=\frac{p^{-1}}{\sqrt{1-n^{-1}}} \sin \sqrt{1-n^{-1} t} \quad$ for $t \in\left[0, \frac{\pi}{\sqrt{1-n^{-1}}}\right]$

$y_{2}^{(n)}(t)=\frac{q^{-1}}{\sqrt{1-n^{-1}}} \sin \sqrt{1-n^{-1}}\left[t+\left(\frac{\pi}{\sqrt{1-n^{-1}}}-\pi\right)\right]$ for $t \in\left[\pi-\frac{\pi}{\sqrt{1-n^{-1}}}, \pi\right]$

See Figure 2.

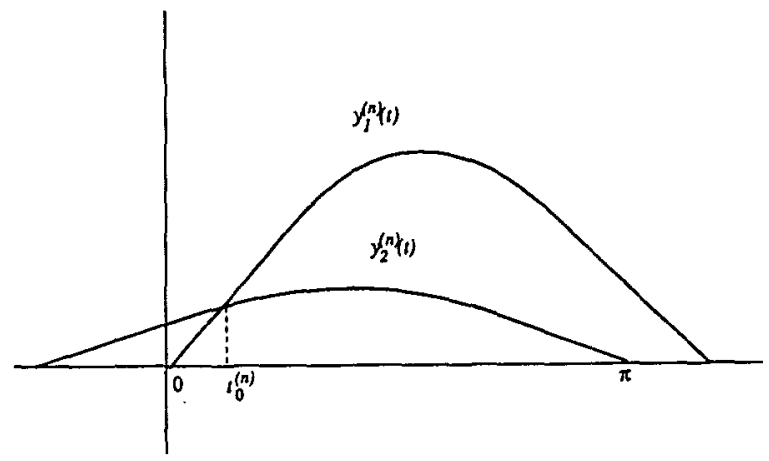

Figure 2

Then there exists a smallest $t_{0}^{(n)} \in(0, \pi)$ such that $y_{2}^{(n)}\left(t_{0}^{(n)}\right)=y_{1}^{(n)}\left(t_{0}^{(n)}\right)$. Form the continuous composite curve:

$$
\widehat{y}_{n}(t)= \begin{cases}y_{1}^{(n)}(t) & \text { if } t \in\left[0, t_{0}^{(n)}\right] \\ y_{2}^{(n)}(t) & \text { if } t \in\left[t_{0}^{(n)}, \pi\right]\end{cases}
$$

Consider now the (non-smooth) $(p, q)$-football of revolution $\widehat{F}_{n}$ defined by $\widehat{y}_{n}(t)$. Clearly, $\sec \left(\widehat{F}_{n}\right) \equiv 1-1 / n$ except at $t=t_{0}^{(n)}$. Rounding off the singularity at $t_{0}^{(n)}$, gives a smooth curve $y_{n}(t)$ whose corresponding $(p, q)$-football of revolution $F_{n}$ $\operatorname{satisfies} \sec \left(F_{n}\right) \geqslant 1-1 / n$. Of course, performing this smoothing process over smaller regions forces the sectional curvature of the resulting $F_{n}$ to blow up at $t_{0}^{(n)}$. This completes the proof.

\section{THEOREM 1}

This section will be devoted to a proof of Theorem 1 . 
Proof of Theorem 1: Let $F$ be a $(p, q)$-football satisfying the hypotheses of Theorem 1. Let

$$
\delta_{0}=\inf \{\delta \geqslant 0 \mid F \text { admits a smooth Riemannian metric with } 0 \leqslant \sec (F) \leqslant 1+\delta\} .
$$

Clearly, $\delta_{0}<+\infty$. We use the notation and setup of $\left({ }^{*}\right)$ with $D=\pi$. Consider the following differential equations:

$$
\begin{aligned}
y^{\prime \prime}+K(t) y & =0, \\
\eta^{\prime \prime}+\left(1+\delta_{0}\right) \eta & =0
\end{aligned}
$$

where $\eta$ is a smooth function with $\eta(0)=0$ and $\eta^{\prime}(0)=y^{\prime}(0)=p^{-1}>0$. Then

$$
\eta(t)=\frac{p^{-1}}{\sqrt{1+\delta_{0}}} \sin \sqrt{1+\delta_{0}} t .
$$

Since by assumption $K(t) \leqslant 1+\delta_{0}$ for $t \in[0, \pi]$, the Sturm Comparison Theorem implies that $y(t) \geqslant \eta(t)$ for $t \in\left[0, \pi / \sqrt{1+\delta_{0}}\right]$.

If $\delta_{0}=0$, then $y(t) \geqslant \eta(t)$ on $[0, \pi]$, and since $y(\pi)=\eta(\pi)=0$, the Sturm theorem implies that $y(t)=\eta(t)$ on $[0, \pi]$. In particular, $y^{\prime}(\pi)=-q^{-1}=\eta^{\prime}(\pi)=-p^{-1}$, and hence $p=q$.

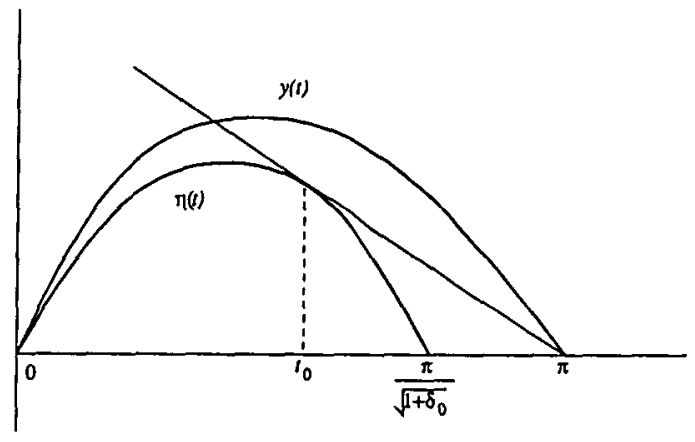

Figure 3

Assume now that $\delta_{0} \neq 0$. Then there exists $t_{0}$ with $\pi /\left(2 \sqrt{1+\delta_{0}}\right)<t_{0}<$ $\pi /\left(\sqrt{1+\delta_{0}}\right)$ such that the tangent line to $\eta$ at $t_{0}$ passes through the point $(\pi, 0)$. See Figure 3. The equation expressing this condition is:

$$
\frac{\left(p^{-1} / \sqrt{1+\delta_{0}}\right) \sin \sqrt{1+\delta_{0}} t_{0}}{t_{0}-\pi}=p^{-1} \cos \sqrt{1+\delta_{0}} t_{0}
$$

This implies that

$$
0=\tan \sqrt{1+\delta_{0}} t_{0}-\sqrt{1+\delta_{0}}\left(t_{0}-\pi\right) \stackrel{\text { def }}{=} \Psi\left(t_{0}\right) .
$$


Now $\Psi\left(\pi / \sqrt{1+\delta_{0}}\right)>0$ and $\Psi\left(\pi /\left(2 \sqrt{1+\delta_{0}}\right)\right)=-\infty$. This suffices to prove the existence of $t_{0}$. Concavity of the function $y(t)$ implies that $-q^{-1}=y^{\prime}(\pi)<\eta^{\prime}\left(t_{0}\right)$ for otherwise $y\left(t_{1}\right)=\eta\left(t_{1}\right)$ for some $t_{1} \in\left[t_{0}, \pi / \sqrt{1+\delta_{0}}\right)$. The Sturm Comparison theorem then implies that $y(t)=\eta(t)$ for $0 \leqslant t \leqslant t_{1}$. Since $y^{\prime \prime} \leqslant 0$, we have $y^{\prime}(t) \leqslant y^{\prime}\left(t_{0}\right)$ for $t \in\left[t_{0}, \pi\right]$. Smoothness of the curve $y$ now implies that $y\left(s_{0}\right)=0$ for some $s_{0} \in\left(t_{1}, \pi\right)$, contradicting $y>0$ on the interval $(0, \pi)$. Hence we may conclude that $-q^{-1}<\eta^{\prime}\left(t_{0}\right)$. This condition is expressed by the equation:

$$
-\frac{1}{q}<\frac{1}{p} \cos \sqrt{1+\delta_{0}} t_{0}
$$

which implies that

$$
\sqrt{1+\delta_{0}} t_{0}<\arccos \left(\frac{-p}{q}\right)
$$

Note that (by the geometrical interpretation of $\left({ }^{* *}\right)$ ), $\delta_{0} \searrow 0$ implies $t_{0} \nearrow \pi$. Thus $\delta_{0}$ is given by the equality case of $\left({ }^{* * *}\right)$. See Remark 12 . Equality in $\left({ }^{* * *}\right)$ combined with condition $\left({ }^{* *}\right)$, yields the desired formula for $\delta_{0}$. This completes the proof.

REMARK 12: The construction in the proof above shows that given $p$ and $q$, there exists a non-smooth $(p, q)$-football $F$ of revolution with $0 \leqslant \sec (F) \leqslant 1+\delta_{0}(p, q)=$ $1+\delta_{0}$ except along a single parallel. Namely, using the notation in the proof of Theorem 1, choose:

$$
y(t)= \begin{cases}\frac{p^{-1}}{\sqrt{1+\delta_{0}}} \sin \sqrt{1+\delta_{0}} t & t \in\left[0, t_{0}\right] \\ -\frac{1}{q}(t-\pi) & t \in\left[t_{0}, \pi\right] .\end{cases}
$$

Note that $y(t)$ is not $C^{2}$ at $t_{0}$.

\section{THEOREM 2}

This section will be devoted to a proof of Theorem 2 .

Proof of Theorem 2: Let $F$ be a $(p, q)$-football satisfying the hypotheses of Theorem 2. Let

$$
\varepsilon_{0}=\inf \left\{\varepsilon \geqslant 0 \mid F \text { admits a smooth Riemannian metric with } 1 \leqslant \sec (F) \leqslant 1+\varepsilon_{0}\right\} .
$$

Clearly, $\varepsilon_{0}<+\infty$. Let $\operatorname{diam}(F)=D$. Applying the Sturm Comparison theorem (using the upper curvature bound) implies that $D \geqslant \pi / \sqrt{1+\varepsilon_{0}}$. The conditions $\left(^{*}\right)$ hold exactly as before. Consider the following differential equations:

$$
\begin{aligned}
\phi^{\prime \prime}+\phi & =0, \\
y^{\prime \prime}+K(t) y & =0, \\
\eta^{\prime \prime}+\left(1+\varepsilon_{0}\right) \eta & =0,
\end{aligned}
$$


where $\eta, \phi$ are smooth functions with $\eta(D)=0$ and $\eta^{\prime}(D)=-p^{-1}$ and $\phi(0)=0$ and $\phi^{\prime}(0)=q^{-1}$. Then

$$
\phi(t)=q^{-1} \sin t
$$

and

$$
\eta(t)=\frac{p^{-1}}{\sqrt{1+\varepsilon_{0}}} \sin \sqrt{1+\varepsilon_{0}}\left(t-\left(D-\frac{\pi}{\sqrt{1+\varepsilon_{0}}}\right)\right) .
$$

Since by assumption $1 \leqslant K(t) \leqslant 1+\varepsilon_{0}$ for $t \in[0, D]$, the Sturm Comparison Theorem implies that

$$
\begin{array}{ll}
\eta(t) \leqslant y(t) & \text { for } t \in\left[D-\frac{\pi}{\sqrt{1+\varepsilon_{0}}}, D\right] \\
y(t) \leqslant \phi(t) & \text { for } t \in[0, D] .
\end{array}
$$

If $\varepsilon_{0}=0$, then $y(t) \geqslant \eta(t)=p^{-1} \sin (t-(D-\pi))$ on $[0, D]$. But $\phi(t) \geqslant \eta(t)$ which implies that $D=\pi$. So, $y(\pi)=\phi(\pi)=0$ and by the equality case of the Sturm comparison theorem we must have $y(t)=\phi(t)$ on $[0, \pi]$, and thus as before we conclude that $p=q$.

We now assume that $\varepsilon_{0}>0$. Obviously, if $\max \eta(t)>\max \phi(t)$, then no such function $y(t)$ can exist. This condition is equivalent to the condition

$$
\frac{p^{-1}}{\sqrt{1+\varepsilon_{0}}}>\frac{1}{q}
$$

which implies that

$$
\varepsilon_{0}<\left(\frac{q}{p}\right)^{2}-1
$$

Hence we can conclude that no $(p, q)$-football of revolution can satisfy $1 \leqslant \sec (F)<$ $(q / p)^{2}$. We now claim that in fact $\varepsilon_{0}=(q / p)^{2}-1$. To see this we proceed as before and construct a non-smooth $(p, q)$-football of revolution with $1 \leqslant \sec (F) \leqslant 1+\varepsilon_{0}=(q / p)^{2}$ at all smooth points. Namely let

$\widehat{y}(t)= \begin{cases}q^{-1} \sin t & \text { if } t \in\left[0, \frac{\pi}{2}\right] \\ \frac{p^{-1}}{\sqrt{1+\varepsilon_{0}}} \sin \sqrt{1+\varepsilon_{0}}\left(t-\left(\frac{\pi}{2}-\frac{\pi}{2 \sqrt{1+\varepsilon_{0}}}\right)\right) \stackrel{\text { def }}{=} \widehat{\eta}(t) & \text { if } t \in\left[\frac{\pi}{2}, \frac{\pi}{2}+\frac{\pi}{2 \sqrt{1+\varepsilon_{0}}}\right]\end{cases}$

See Figure 4.

Note that $\hat{\eta}$ is just $\eta$ with $D=\pi / 2+\pi /\left(2 \sqrt{1+\varepsilon_{0}}\right)$. Let $\widehat{F}$ be the football generated by $\widehat{y}(t)$, then $\operatorname{diam} \widehat{F}=\pi / 2+\pi /\left(2 \sqrt{1+\varepsilon_{0}}\right)$. Since any smooth football $F$ with $\operatorname{diam} F=\operatorname{diam} \widehat{F}$ has a generating curve $y$ which would have to pass through the 


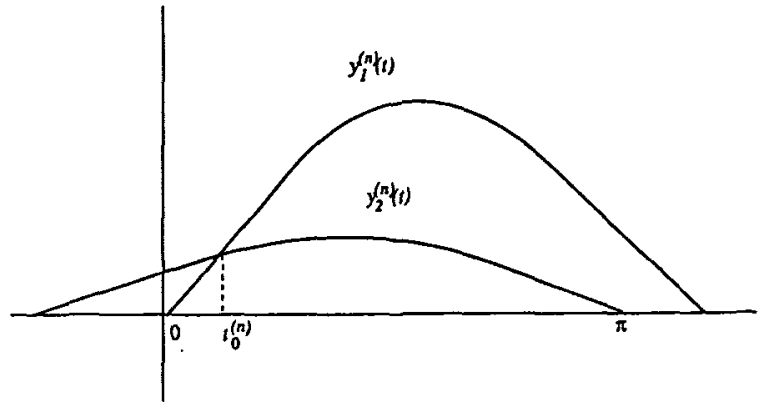

Figure 4

point $(\pi / 2, \widehat{y}(\pi / 2))$, the equality case of the Sturm comparison theorem would imply that $y$ would agree with $\phi(t)$ on $[0, \pi / 2]$ and $\widehat{\eta}(t)$ on $[\pi / 2, \operatorname{diam} \widehat{F}]$, which would contradict the smoothness of $y(t)$. So now suppose that $D=\operatorname{diam} F \neq \operatorname{diam} \widehat{F}$. Note that changing $D$ amounts to a simple horizontal translation of $\widehat{\eta}(t)$ and any such translation $\eta$ would attain a maximum of $q^{-1}$ at $t=t_{0} \neq \pi / 2$. But then $\eta\left(t_{0}\right)>\phi\left(t_{0}\right)$, and as we have seen before, no generating function $y(t)$ would exist. This completes the proof.

\section{The Optimal Pinching Constants}

In this section we tabulate some values of the optimal pinching constants $\delta_{0}(p, q)$ and $\varepsilon_{0}(p, q)$, for special values of $p / q$, and also give the graph of $\delta_{0}$ as a function of $p / q$.

\begin{tabular}{|c||c|c|}
\hline$p / q$ & $\delta_{0}(p, q)$ & $\varepsilon_{0}(p, q)$ \\
\hline \hline .01 & 1044.4 & 9999.0 \\
\hline .03 & 122.55 & 1110.1 \\
\hline .05 & 46.254 & 399.00 \\
\hline .07 & 24.588 & 203.08 \\
\hline .09 & 15.411 & 122.46 \\
\hline .10 & 12.683 & 99.000 \\
\hline .20 & 3.5092 & 24.000 \\
\hline .30 & 1.5893 & 10.111 \\
\hline .40 & 0.8505 & 5.2500 \\
\hline .50 & 0.4835 & 3.0000 \\
\hline
\end{tabular}

\begin{tabular}{|c||c|c|}
\hline$p / q$ & $\delta_{0}(p, q)$ & $\varepsilon_{0}(p, q)$ \\
\hline \hline .60 & 0.2752 & 1.7778 \\
\hline .70 & 0.1482 & 1.0408 \\
\hline .80 & 0.0689 & 0.5625 \\
\hline .90 & 0.0213 & 0.2346 \\
\hline .93 & 0.0120 & 0.1562 \\
\hline .95 & 0.0071 & 0.1080 \\
\hline .97 & 0.0032 & 0.0628 \\
\hline .99 & 0.0006 & 0.0203 \\
\hline 1.0 & 0.0000 & 0.0000 \\
\hline
\end{tabular}

The graph of $\delta_{0}$ as a function of $p / q$ is given in Figure 5 . 


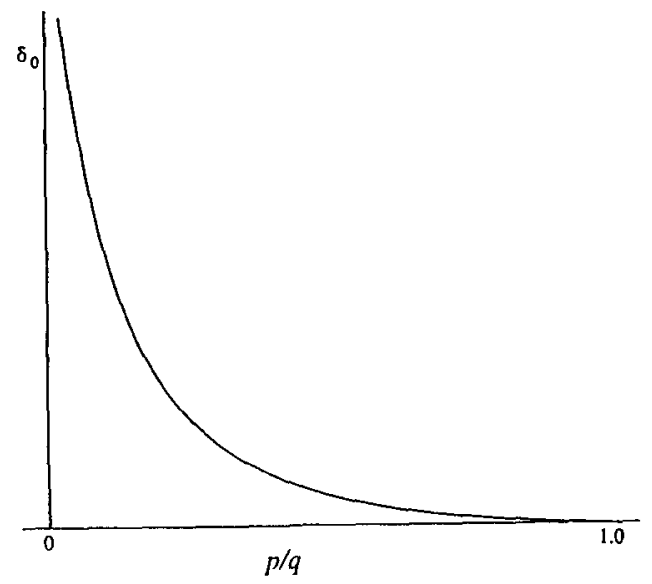

Figure 5

\section{REFERENCES}

[1] J. Borzellino, Riemannian geometry of orbifolds, Ph.D thesis (University of California, Los Angeles, 1992).

[2] J. Borzellino, 'Orbifolds of maximal diameter', Indiana Univ. Math. J. 42 (1993).

[3] B. O'Neill, Elementary differential geometry (Academic Press, New York, 1966).

[4] M. Spivak, A comprehensive introduction to differential geometry Vol. IV (Publish or Perish, Delaware, 1979).

[5] W. Thurston, The geometry and topology of 3-manifolds, Lecture Notes (Princeton University Math. Dept., 1978).

Department of Mathematics

University of California

Davis CA 95616

United States of America 